\title{
Numerical sampling rules for paraxial regime pulse diffraction calculations
}

\author{
Damien P. Kelly, ${ }^{1, *}$ Bryan M. Hennelly, ${ }^{2}$ Alexander Grün, ${ }^{1}$ and Karl Unterrainer ${ }^{1}$ \\ ${ }^{1}$ Institut für Photonik und Zentrum für Mikro- und Nanostrukturen, Technische Universität Wien, \\ A-1040 Wien, Austria \\ ${ }^{2}$ Department of Computer Science, National University of Ireland, Maynooth, Ireland \\ *Corresponding author: damienpkelly@gmail.com
}

Received March 10, 2008; revised June 6, 2008; accepted June 17, 2008; posted July 9, 2008 (Doc. ID 93606); published August 19, 2008

\begin{abstract}
Sampling rules for numerically calculating ultrashort pulse fields are discussed. Such pulses are not monochromatic but rather have a finite spectral distribution about some central (temporal) frequency. Accordingly, the diffraction pattern for many spectral components must be considered. From a numerical implementation viewpoint, one may ask how many of these spectral components are needed to accurately calculate the pulse field. Using an analytical expression for the Fresnel diffraction from a 1-D slit, we examine this question by varying the number of contributing spectral components. We show how undersampling the spectral profile produces erroneous numerical artifacts (aliasing) in the spatial-temporal domain. A guideline, based on graphical considerations, is proposed that determines appropriate sampling conditions. We show that there is a relationship between this sampling rule and a diffraction wave that emerges from the aperture edge; comparisons are drawn with boundary diffraction waves. Numerical results for 2-D square and circular apertures are presented and discussed, and a potentially time-saving calculation technique that relates pulse distributions in different $z$ planes is described. (C) 2008 Optical Society of America

OCIS codes: $320.5550,070.0070,070.2025,070.7345,030.0030$.
\end{abstract}

\section{INTRODUCTION}

Ultrashort and few-cycle pulses are finding increasing application in modern optics, particularly as new femtosecond ( $f$ s) sources become more reliable and affordable. These pulses are in common use in areas such as optical coherence tomography, confocal microscopy, terahertz $(\mathrm{THz})$ generation and detection, and spectroscopy [1-19]. Pulses generated in fs sources tend to have a broad spectrum $(\sim 20 \mathrm{~nm})$ consisting of many different wavelengths and so differ from the approximately monochromatic light produced by most standard lasers. In general, there is a complicated relationship between different spectral components. Several authors have examined how these pulses propagate and diffract by using analytical methods [1-10]. It has been shown that different spectral components do not diffract in the same manner, and so these pulses can exhibit dispersion in free-space propagation [10]. Furthermore, several types of pulses exist that exhibit different spectral characteristics on propagation $[1-4,10,11,13]$.

To calculate the diffraction pattern of these pulses in space and time, one must first consider the spatial diffraction pattern formed by an individual spectral component. Under paraxial conditions, the diffracted field due to a single spectral component may be described using the Fresnel transform (FST) [20,21]. This calculation is repeated for each spectral component, giving a spatialspectral distribution. The diffracted spatial-temporal pulse is then found by performing an inverse Fourier transform (with respect to the temporal frequency) on this spatial-spectral distribution $[11,13,14]$. In some cases, analytical expressions describing the spatial- temporal distribution exist [13], however, in order to analyze general diffraction problems, it is important to develop complementary numerical techniques $[15,16,22]$. Since many different spectral components contribute to a pulse's diffraction pattern, a fast numerical technique for calculating the diffraction pattern formed by a single wavelength is clearly important. In previous publications we have shown how the linear canonical transform (LCT) may be implemented using a fast Fourier transform (FFT) approach [22-25]. The LCT is a general transform that includes the FST as a special case. Using ideas outlined in $[23,24]$, we have examined the numerical implementation of the Fresnel transform and shown that both the magnitude and phase of the distribution can be accurately calculated [22,25].

In this paper we examine how many spectral components need to be considered in order to calculate a pulse diffraction pattern free of (spatial and temporal) aliasing. We approach this in the following manner:

(i) In Section 2 we carry out a theoretical analysis (1-D) and derive sampling guidelines that allow the field of a pulse diffracted by a 1-D slit to be calculated without aliasing. These sampling guidelines consider the temporal sampling rate, temporal frequency and time extents, the size of the diffracting aperture (1-D slit), and the spatial extent in the diffraction plane, as well as the distance of the diffraction plane from the aperture.

(ii) In Section 3 numerical results are presented that support the 1-D analysis from Section 2. These numerical results are calculated for the 2-D case using an FFT-based implementation of the FST $[22,25]$.

(iii) In Section 4 we examine a numerical redundancy 
that allows the pulse diffraction pattern at two different distances from the aperture to be related.

(iv) In Section 5 our sampling guidelines are examined using the method of stationary phase and are shown to be related to a diffraction wave that emerges from "critical points" located at the aperture edge.

(v) Finally, in Section 6 a brief conclusion is presented.

\section{THEORETICAL ANALYSIS}

We begin by presenting a 1-D analysis $(x)$ and assume that the paraxial approximation is valid. We further assume that the pulse filling the aperture has a spatially invariant spectrum at that plane $(z=0)$-corresponding to a Type I pulse as described in $[10,11]$. Under these conditions, we define the sampling rules that allow accurate implementation of our numerical technique. Following the approach outlined by $\mathrm{Gu}$ et al. [6,11] and others $[13,14]$, we write the diffracted distribution for an individual spectral component using the FST as

$$
\tilde{U}_{K}(x, K)=U_{K}(x, K) \exp \left(\frac{j z \omega}{c}\right),
$$

with

$$
U_{K}(x, K)=\sqrt{\frac{1}{j K}} \int_{-\infty}^{\infty} u(X) p(X) \exp \left[\frac{j \pi}{K}(x-X)^{2}\right] \mathrm{d} X,
$$

and where $c$ is the speed of light, $K=(\lambda z)=(2 \pi z c) / \omega, \lambda$ is the wavelength of the particular spectral component, $z$ is the propagation distance, $\omega$ is the temporal frequency of the light, $p(\cdot)$ is the aperture function, and $u(x)$ is the spatial distribution of the field incident at the aperture. Assuming the pulse to have a temporal frequency distribution given by $V(\omega)$, we can write the spatial-spectral distribution as

$$
U(x, K)=\widetilde{U}_{K}(x, K) V(\omega) .
$$

Finally, the spatial-temporal distribution can be found by evaluating the integral $[13,14,19]$

$$
U\left(x, t_{R}, z\right)=\int_{0}^{\infty} U_{K}(x, K) V(\omega) \exp \left(-j \omega t_{R}\right) \mathrm{d} \omega
$$

where we have introduced the retarded time $t_{R}[15,16]$,

$$
t_{R}=t-z / c .
$$

Equations (1)-(3) are the analytical equations describing the diffraction process. By introducing the retarded time, we cancel the effect of the linear phase term on the righthand side of Eq. (1a) $[15,16,22]$. Using the retarded time is also advantageous from a numerical point of view, since removing the linear phase term can greatly reduce the number of samples required to represent the signal $[15,16,22]$. Also, we note that with $z=0$, the time $t_{R}=t=0$ refers to that instant when the peak pulse amplitude fills the aperture. If $p(x)$ is given by

$$
p(x) \equiv\left\{\begin{array}{ll}
1, & |x|<L \\
0, & |x|>L
\end{array},\right.
$$

and assuming uniform illumination, we set $u(x)=1$ and solve Eq. (1b) to give [22,26]

$$
\begin{aligned}
U_{K}(x, K)= & -\frac{1}{2}\left\{\operatorname{erf}\left[\sqrt{\frac{\pi}{K}}(-1)^{3 / 4}(L-x)\right]\right. \\
& \left.+\operatorname{erf}\left[\sqrt{\frac{\pi}{K}}(-1)^{3 / 4}(L+x)\right]\right\} .
\end{aligned}
$$

We are interested in calculating Eq. (3a) numerically using a finite number of contributing spectral components, $N_{\omega}$; therefore we now write the discrete form of Eq. (3a) [27-30] as

$$
U_{S}\left(x, t_{R}, z\right)=\sum_{n=1}^{N_{\omega}} U_{K}\left(x, K_{n}\right) V\left(\omega_{n}\right) \exp \left(-j \omega_{n} t_{R}\right),
$$

where the subscript $S$ distinguishes between the ideal analytical signal $U$ and its sampled counterpart, which is calculated using the vectors $K_{n}=\left[K_{1}, K_{2}, \ldots, K_{N \omega}\right]$ and $\omega_{n}$ $=\left[\omega_{1}, \omega_{2}, \ldots, \omega_{N \omega}\right]$. There are significant differences between these two representations. For one, we expect following sampling theory [27-30] that the discretization process will produce an infinite number of replicas in the time domain that (i) are repeated periodically and where (ii) the separation between them is related by $N_{\omega}$ [and indeed to the spectral frequency extent $\left(\omega_{N \omega}-\omega_{1}\right)$, which is discussed in more detail later in Section 2]. If we wish to recover our signal we need to ensure that $N_{\omega}$ is sufficiently large so that successive replicas do not overlap. However, as we noted in the Introduction, it is not sufficient to consider the $\omega$ and $t_{R}$ variables, as the spatial variable $x$ also plays a role. Before we move on to consider how changing $x$ affects the sampling conditions, let us first consider how to sample the pulse spectrum, $V(\omega)$, sufficiently at the aperture plane.

We begin by introducing the concept of the timefrequency (temporal) product (TFP) [17,18,31-33]. Consider the temporal signal, $g(t)$. If the energy of $g(t)$ is localized within some finite region in both the time and temporal-frequency domains, we can write [31-33]

$$
\int_{-\Delta \omega / 2+\omega_{0}}^{\Delta \omega / 2+\omega_{0}}|\widetilde{g}(\omega)|^{2} \mathrm{~d} \omega=\int_{-\Delta t / 2}^{\Delta t / 2}|g(t)|^{2} \mathrm{~d} t=\eta_{\omega} E_{\omega}
$$

where $E_{\omega}$ is the energy contained over the entire temporal spectrum and $\eta_{\omega} \sim 1$. In Eq. (5a), it is assumed that $g(t)$ and $\widetilde{g}(\omega)$ are centered at the coordinates $t=0$ and $\omega=\omega_{0}$, respectively. Using uniform sampling steps, the number of samples required to represent $g(t)$ is given by the TFP [23-25,31-33],

$$
N_{\omega}=\frac{\Delta t \Delta \omega}{4 \pi} .
$$

Let us assume a Gaussian pulse of the form [11] 


$$
V(\omega)=\sqrt{T} \exp \left\{-\left[\frac{T\left(\omega-\omega_{0}\right)}{\sqrt{2}}\right]^{2}\right\},
$$

with a corresponding Fourier transform (with respect to the temporal frequency)

$$
v(t)=\frac{1}{\sqrt{T}} \exp \left(-j \omega_{0} t\right) \exp \left[-\left(\frac{t}{\sqrt{2} T}\right)^{2}\right] .
$$

The parameter $T$ is related to the width of the Gaussian pulse in the time and temporal frequency domains [11]; for example, in the time domain, the full width at halfmaximum is given by $2 \sqrt{2 \ln (2)} T$ and $\omega_{0}$ is the central frequency of the pulse. It can be shown that $99 \%$ of the energy in the Gaussian pulse is contained between $-4 T<t$ $<4 T$ in the time domain and between $-4 / T+\omega_{0}<\omega$ $<4 / T+\omega_{0}$ in the frequency domain. Applying these energy considerations, we define our temporal frequency extent as $\Delta \omega=8 / T$. We wish to ensure that successive replicas do not overlap, and therefore we propose that $N_{\omega}$ be chosen such that the time extent of the signal, $\Delta t=1 / \delta \omega$ $=40 \mathrm{~T}$. We note that this choice is arbitrary; however, from experience we have found that it is suitable for most of the numerical simulations we have encountered [22,25]. With uniform sampling, $N_{\omega}$ is thus given by the TFP,

$$
N_{\omega}=\frac{\Delta t \Delta \omega}{4 \pi}=\frac{1}{4 \pi}(40 T)\left(\frac{8}{T}\right)=\frac{80}{\pi},
$$

and

$$
\Delta t=(\delta \omega)^{-1}=\frac{N_{\omega}}{\Delta \omega}(4 \pi) .
$$

Equations (7a) and (7b) define the guidelines for sampling our Type-I pulse at the aperture plane. We now wish to examine the pulse distribution in the diffraction plane. So, substituting Eqs. (6a) and (4b) into Eq. (4c) and following the sampling guidelines for $V(\omega)$ from Eqs. (7a) and (7b), we calculate $\left|U_{S}\left(x, t_{R}, z\right)\right|$ using a trapezoidal numerical integration technique [34] and plot the results in Fig. 1. The simulation values are as follows: $T=0.5$ $\times 10^{-12} \mathrm{~s}, z=1 \mathrm{~m}, \omega_{0}=1.6 \times 10^{12} \mathrm{rad} \mathrm{s}^{-1}$, and $L=25 \mathrm{~mm}$. In these numerical results we can see two replicas: the first at $t_{R}=0$ and the second at $t_{R}=\Delta t / 2=20 \mathrm{~T}$. The pulse's energy spreads out into four individual tails (see Fig. 1) that trail behind each pulse replica. These residual tails overlap with the other sampled replicas (see Fig. 1), which causes aliasing in the calculated distribution. Although we have presented only one specific case, we have performed these numerical calculations for a variety of different values of $z$ and $\omega$ and believe this is a representative example (see figure caption for details about the range of the contour plot). The task we now set ourselves is to find a relationship between $x$ and $t_{R}$ that allows us to predict where the trailing tails of one replica intercept the following replica, i.e., for a given time $t_{R}=\Delta t / 2$, identify the point $\left(x_{\mathrm{SL}}, t_{R}\right)$ that marks the onset of aliasing. Since the form of Eq. (4c) is relatively difficult to manipulate, we seek a simplier expression that describes the path of these trailing tails. To this end we are going to change

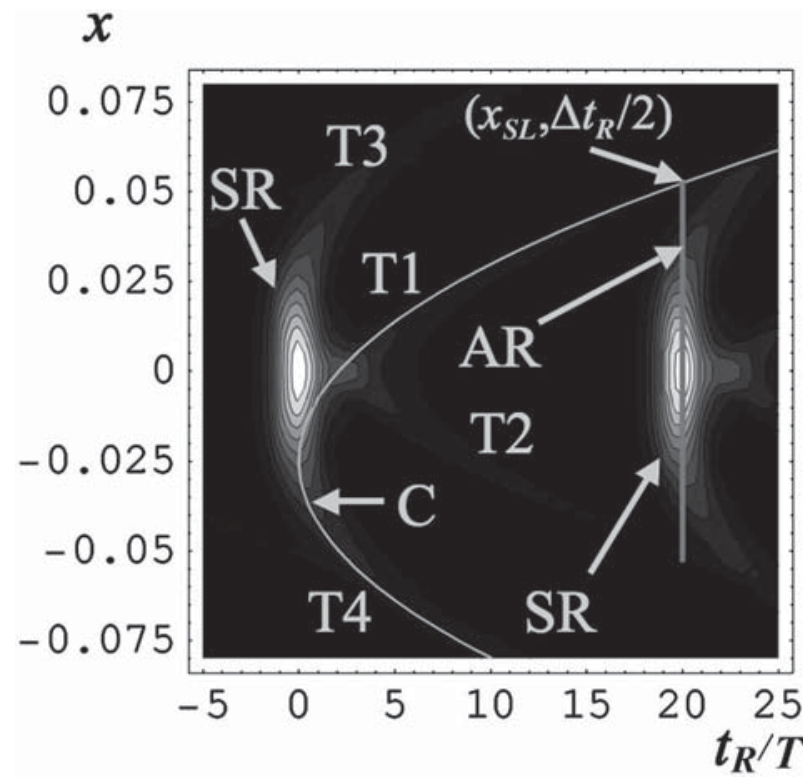

Fig. 1. Contour plot of $\left|U_{S}\left(x, t_{R}, z=1\right)\right|$ for $-0.075 \mathrm{~m}<x$ $<0.075 \mathrm{~m}$ and $-5<t_{R} / T<25$. There are ten contour levels spanning the range $0<\left|U_{S}\left(x, t_{R}, z=1\right)\right|<3.56 \times 10^{6}$. T1, T2, T3, T4, pulse tails 1-4; AR, aliasing free region delimited by $\left(x_{\mathrm{SL}}, t_{R}\right) ; \mathrm{SR}$, sampled replica; C, curve plotted using Eq. (9b).

some of the equations we introduced earlier. We are going to replace the $p(\cdot)$ [as defined in Eq. (4a)] with $p_{T}(\cdot)$, which we define as

$$
p_{T}(x) \equiv \delta(x-L)+\delta(x+L)
$$

where $\delta$ is the Dirac delta function [20,27]. With this simplification, Eqs. (4b) and (3a), (note: $U_{K} \rightarrow \psi_{K}$ and $U \rightarrow \psi$ ) now become

$$
\psi_{K}(x, K)=\sqrt{\frac{1}{j K}}\left\{\exp \left[\frac{j \pi}{K}(x-L)^{2}\right]+\exp \left[\frac{j \pi}{K}(x+L)^{2}\right]\right\}
$$

and

$$
\begin{aligned}
\psi\left(x, t_{R}, z\right)= & \mathfrak{I}^{-1}\left\{V(\omega)\left[\exp \left(j \omega R_{1}\right)+\exp \left(j \omega R_{2}\right)\right]\right\}\left(t_{R}\right) \\
= & \mathfrak{I}^{-1}\left\{V(\omega) \exp \left(j \omega R_{1}\right)\right\}\left(t_{R}\right)+\mathfrak{I}^{-1}\left\{V(\omega) \exp \left(j \omega R_{2}\right)\right\} \\
& \times\left(t_{R}\right)=v\left(t_{R}-R_{1}\right)+v\left(t_{R}-R_{2}\right),
\end{aligned}
$$

where $\mathfrak{I}^{-1}\{g(\omega)\}\left(t_{r}\right)=\int_{0}^{\infty} g(\omega) \exp \left(-j \omega t_{r}\right) \mathrm{d} \omega$ indicates the inverse (temporal) Fourier transform operator [19], $R_{1}=(x$ $-L)^{2} / 2 z c, R_{2}=(x+L)^{2} / 2 z c$ and where we have used Eqs. (6a) and (6b) and invoked the Fourier shift theorem [27]. Since we specifically wish to examine the effects of sampling, we may also calculate Eq. (8c) numerically by calculating the discrete Fourier transform [27]

$$
\begin{aligned}
\psi_{S}\left(x, t_{R}, z\right)= & \sum_{n=1}^{N_{\omega}}\left[\exp \left(j \omega_{n} R_{1}\right)+\exp \left(j \omega_{n} R_{2}\right)\right] V\left(\omega_{n}\right) \\
& \times \exp \left(-j \omega_{n} t_{R}\right) .
\end{aligned}
$$

Equations (8b)-(8d) are easier to analyze and appear to exhibit many of the characteristics of the previous case. In Fig. 2, we present a plot of $\left|\psi_{S}\right|$ [Eq. (8d)] generated us- 


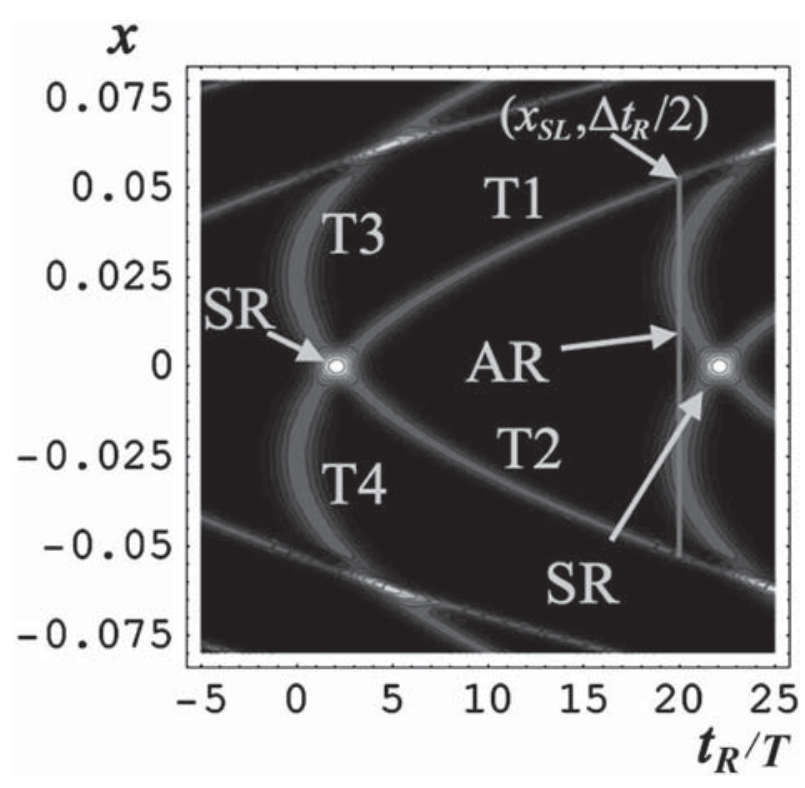

Fig. 2. Contour plot of $\left|\psi_{S}\right|$ for $-0.075 \mathrm{~m}<x<0.075 \mathrm{~m}$ and -5 $<t_{R} / T<25$. There are ten contour levels spanning the range 0 $<\left|\psi_{S}\right|<323 \times 10^{3}$. T1, T2, T3, T4, pulse tails $1-4 ; \mathrm{AR}$, aliasing free region delimited by $\left(x_{\mathrm{SL}}, t_{R}\right)$; SR, sampled replica.

ing the same parameters as those used to produce Fig. 1 from Eq. (4c), (see figure caption for contour details). Again we can see two replicas, each of which has four trailing tails; see Fig. 2. The form of the trailing tails appears to be similar to those found in Fig. 1. In particular, the intersection point of Tail 1 (T1) with the following replica occurs at approximately the same value of $x$ in both figures. Clearly, however, there are several differences between the two figures. For example, in Fig. 2 the maximum magnitude remains constant as one travels along the tail path. Whereas in Fig. 1, the magnitude of the tails decrease as $x$ and $t_{R}$ increase. This energy appears to be redistributed from the tails toward the center of the pulse. There are also differences in the maximum magnitude of $\left|\psi_{S}\right|$ and $\left|U_{S}\left(x, t_{R}, z\right)\right|$, which we partly attribute to differences in the aperture functions; see Eqs. (4a) and (8a). Again we draw the readers' attention to the similarity of the paths taken by the trailing tails in Figs. 1 and 2 and propose that the simplified relationship in Eqs. (8c) and $(8 \mathrm{~d})$ be used to determine the conditions under which aliasing occurs. We proceed by examining Eq. (8d) with a view to determining a relationship between $x$ and $t_{R}$ that defines the path of these trailing tails. For all the cases we have examined, the function $v(\cdot)$ has a maximum value when the argument is zero. Assuming this is always true, we find that the first (second) term in Eq. (8a) will have a maximum when $t_{R}=R_{1}\left(t_{R}=R_{2}\right)$. Substituting $x, z$, and $L$ in for $R_{1}$ and $R_{2}$, we find that

$$
x= \pm \sqrt{2 z c t_{R}}+L \quad \text { for } R_{1}
$$

and

$$
x= \pm \sqrt{2 z c t_{R}}-L \quad \text { for } R_{2} .
$$

The relationship between Eq. (9) and the tails predicted by the numerical integration of Eq. (4c) was further ex- amined. The location (in $x$ ) of the maximum values of $\left|U_{S}\left(x, t_{R}, z\right)\right|$, [numerical search values (NSV)], over the region $0<t_{R}<25$, were found using a numerical search algorithm. These results were overlayed on Eq. (9b) in Fig. 3 . The locations of maximum values of $\left|U_{S}\left(x, t_{R}, z\right)\right|$ clearly do not fall exactly on the curve predicted by Eq. (9b), rather they appear to be slightly displaced (see Fig. 3). Nevertheless, the locations of the maximum points seem to be related to Eq. (9b), and the displacement between the curve and the maximum points remains approximately constant for $4<t_{R}<18$. For $t_{R}>18$ the NSV are located on axis $(x=0)$ due to the presence of the second pulse replica, which can be seen by comparing Figs. 3 and 1. Also, from Fig. 1 we note that the curve $C$ predicted by Eq. (9b) clearly follows the path taken by Tail 1 .

With Eq. (9), we now have an analytical expression describing the path taken by the tails, which trail each pulse replica. Aliasing will occur when the tails from one replica overlap with a subsequent replica. The spacing in time between these successive replicas is determined by Eq. (7); for example, the time $t_{R}=\Delta t / 2$ marks the arrival of the second replica's peak pulse amplitude. In order to discuss aliasing in the pulse distribution about this peak amplitude point, we introduce the variable $t_{v}$ setting $t_{R}$ $=\left(\Delta t / 2+t_{v}\right)$ in Eq. (9a) and (9b). Then, substituting Eq. (7) into Eq. (9), we can identify a region in space and time [delimited by the point $x_{\mathrm{SL}}\left(t_{v}\right)$ ], where the pulse can be calculated without the effects of aliasing. This region is given by

$$
x_{S L 1}\left(t_{v}\right)= \pm \sqrt{2 z c\left(\frac{2 \pi N_{\omega}}{\Delta \omega}+t_{v}\right)}+L \quad \text { for } R_{1}
$$

and

$$
x_{S L 2}\left(t_{v}\right)= \pm \sqrt{2 z c\left(\frac{2 \pi N_{\omega}}{\Delta \omega}+t_{v}\right)}-L \quad \text { for } R_{2}
$$

Later in Section 3, we present numerical results showing the arrival of the pulse's peak amplitude at $t_{v}=0$ and also

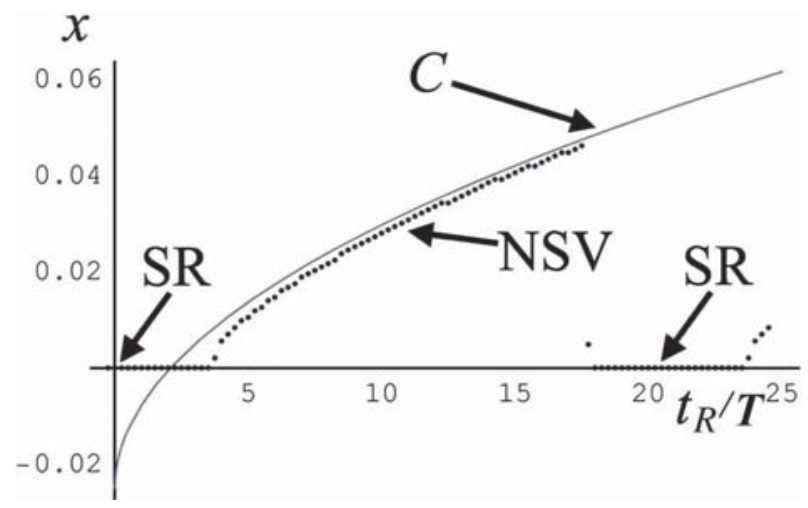

Fig. 3. Plot of Eq. (9b) with $L=25 \mathrm{~mm}$. SR, sampled replica; $\mathrm{NSV}$, numerical search values (these values were found by performing a maximum value numerical search of $\left|U_{S}\left(x, t_{R}, z=1\right)\right|$ over the range $-0<x<0.07 \mathrm{~m}$ and $0<t_{R} / T<25$ ). 
just before this time, i.e., $t_{v}<0$. We note the following results from Eq. (10): (i) $x_{\mathrm{SL}}$ varies as a function of $t_{v}$ and (ii) the aliasing free region delimited by $x_{\mathrm{SL}}$ may be made larger by increasing $N_{\omega}$; however, this increase occurs at a rate of $\sqrt{N_{\omega}}$.

There are several issues that still remain: The analysis presented was for the 1-D case and for a Gaussian spectral profile, $V(\omega)$. Nevertheless, we believe that these sampling rules extend to the 2-D case $(x$ and $y)$ and are also suitable for different types of spectral profiles. In the next section we thus present numerical results for the 2-D case where a non-Gaussian (Type-I) spectral distribution describes the pulse's spectral profile. We show that the theoretical conclusions arrived at in this section also appear to be valid for this more general case.

The sampling rules proposed in this section are derived using the relatively simple formulas, Eqs. (8a)-(8d), to describe the pulse tails that arise in the diffraction process.
Later in Section 5 we will show that the main contributions to our diffraction integral, Eq. (1), come from specific points located at the aperture edge, and we comment on the similarity between this and the predictions of geometrical diffraction and boundary wave diffraction [36-39].

\section{NUMERICAL RESULTS}

In this section we present some numerical results for the 2-D diffraction pattern formed when a pulse is incident upon either a square or a circular aperture. We have already mentioned that we are going to examine the diffraction process numerically for a spectral profile that is no longer Gaussian in shape to see whether the results derived in Section 2 are also applicable here. So from [14] we now assume that our spectral distribution is given by

$$
V_{2}(\omega)=\left\{\begin{array}{cc}
\left(\frac{s+1}{\omega_{b}}\right)^{s+1} \frac{\left(\omega-\omega_{a}\right)^{s}}{\Gamma(s+1)} \exp \left[-(s+1) \frac{\omega-\omega_{a}}{\omega_{b}}\right], & \text { for } \omega>\omega_{a} \\
0, & \text { otherwise }
\end{array}\right.
$$

where $\Gamma$ is the gamma function $[14,35]$.

In Fig. 4 we present a plot of Eq. (11) (see solid line), with the following values: $s=1, \omega_{b}=6 \times 10^{12}$, and $\omega_{a}=100$ $\times 10^{12}$. To ensure this plot has a maximum value of unity, we have divided Eq. (11) by its maximum value of 0.37 . We have also plotted a normalized Gaussian pulse, calculated using Eq. (7a) with $\omega_{0}=110 \times 10^{12}$ and $T=0.35$ $\times 10^{-12}$. We normalize the plots merely as an aid to the reader so that it is easier to visually compare both distributions. In line with our sampling guidelines, the Gaussian pulse is plotted over the range $-4 / T+\omega_{0}<\omega<4 / T$ $+\omega_{0}$. We have been careful with our choice of parameters ( $T$ and $\omega_{0}$ ) for the Gaussian pulse, ensuring that the resulting distribution overlaps with $V_{2}(\omega)$. One can see from the $V_{2}(\omega)$ distribution that more spectral energy is contained at lower frequencies. Since our sampling rules merely state choose $\Delta \omega=8 / T$ and $N_{\omega}$ (such that $\Delta t=40 T$ ), the asymmetry of $V_{2}(\omega)$ has no bearing on the aliasing conditions derived in Eq. (10).

In the following numerical results we have set $z=0.1$, chosen a circular aperture of radius, $L$, and a square aperture with a total width of $2 L$, where $L=0.1 \mathrm{~mm}$. We make two more comments about these results before we present them.

(i) Because we specifically wish to examine conditions under which aliasing occurs, we have purposely broken our own sampling guidelines, choosing $N_{\omega}=20$ to emphasis any numerical artifacts that occur.

(ii) The second comment relates to the manner in which we have calculated the Fresnel integral. To gain insight into the pulse diffraction process, previously we used an analytical expression for the diffraction integral. However, in many cases an analytical solution may not exist, and so fast methods for numerically implementing the Fresnel transform accurately are required. In previous publications we have outlined how these calculations may be performed, and we now use these techniques to calculate the diffracted pulse field [22-25].

In Figs. 5(a) and 5(b) we present the diffraction pattern of a pulse (3-D and contour) after it has been incident upon a square aperture with time $t_{v}=0$. Aliasing effects are quite noticeable in the $3-\mathrm{D}$ plot, and attention is drawn to them in the contour plot (Fig. 5(b)), using a gray box (red online). This box is calculated using Eq. (10). We choose that value of $x_{\mathrm{SL}}$ from Eqs. (10a) and (10b) that minimizes the aliasing free region. The coordinates of the box, $\{x, y\}$ starting in the top left corner and moving clockwise are given by $\left\{\left\{-x_{\mathrm{SL}}, x_{\mathrm{SL}}\right\},\left\{x_{\mathrm{SL}}, x_{\mathrm{SL}}\right\},\left\{x_{\mathrm{SL}},-x_{\mathrm{SL}}\right\}\right.$, and $\left\{-x_{\mathrm{SL}},-x_{\mathrm{SL}}\right\}$. Inspecting Fig. $5(\mathrm{~b})$, it can be seen that the aliasing rules suggested by Eq. (10) accurately predict the location where aliasing occurs. In Figs. 6(a) and 6(b) we present the same plots, this time, however, for a circular aperture. The peak amplitude of the pulse is lower than in Fig. 5(a), which we attribute to differences in the size of the contributing areas of the respective apertures. The contrast in area between the two cases, $\pi L^{2}$ versus $(2 L)^{2}$, is directly related to the amount of energy that is admitted into the system $[11,20,26]$. Aliasing is also present in the circular aperture plots; however, the magnitude is lower than in Fig. 5(a) and the numerical artifacts introduced are symmetric, forming a circle about the center pulse with a radius $x_{\mathrm{SL}}$. Again it appears that the rules in Eq. (10) apply. This can be seen in Fig. 6(b). [We note that these results have been plotted in Mathematica, where for Fig. 6(b), we have set the "PlotRange" function to "Automatic" [35], which means that the ten contour levels 


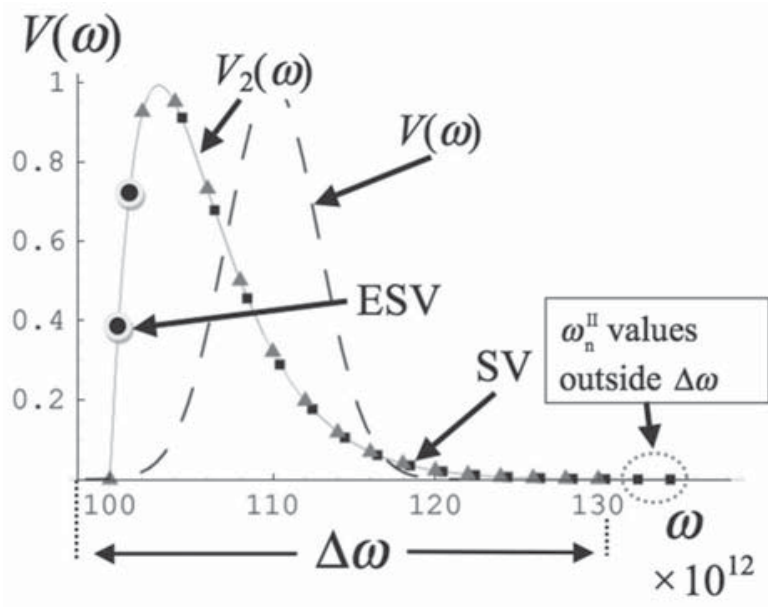

Fig. 4. Comparison of $V(\omega)$ and $V_{2}(\omega)$, where $\omega \times 10^{12} \mathrm{rad} \mathrm{s}^{-1}$. SV, sampled values; ESV, extra sampled values. For Section 4 , gray triangles indicate the SV for plane $z_{1}$, black boxes indicate the SV for plane $z_{2}$, and ESV represent the two extra sampled points necessary for diffraction pattern calculation at $z_{2}$.

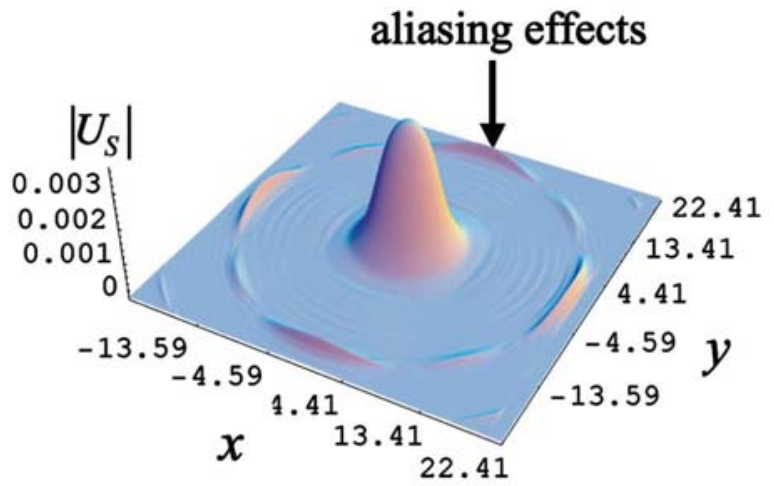

(a)

$$
x
$$

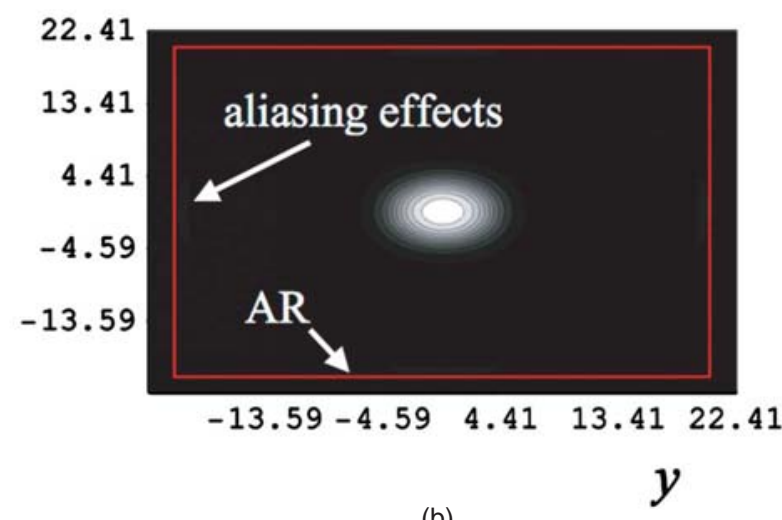

Fig. 5. (Color online) (a) Numerical results of $\left|U_{S}\right|$ for a square aperture, with $t_{v}=0 \mathrm{~s}, z=0.1 \mathrm{~m}$, and $L=0.1 \mathrm{~mm}$ over the range $-23 \mathrm{~mm}<x<23 \mathrm{~mm},-23 \mathrm{~mm}<y<23 \mathrm{~mm}$. (b) Contour plot of $\left|U_{S}\right|$ for a square aperture, with $t_{v}=0 \mathrm{~s}, z=0.1 \mathrm{~m}$, and $L$ $=0.1 \mathrm{~mm}$ over the range $-23 \mathrm{~mm}<x<23 \mathrm{~mm},-23 \mathrm{~mm}<y$ $<23 \mathrm{~mm}$. Coordinates of the gray box (red online) defined in text, with $x_{\mathrm{SL}}=20.3126 \mathrm{~mm}$. AR, aliasing region.

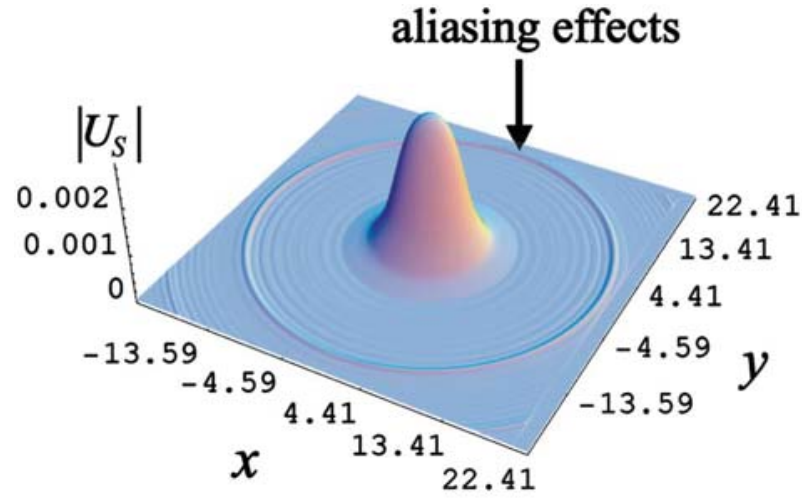

(a)

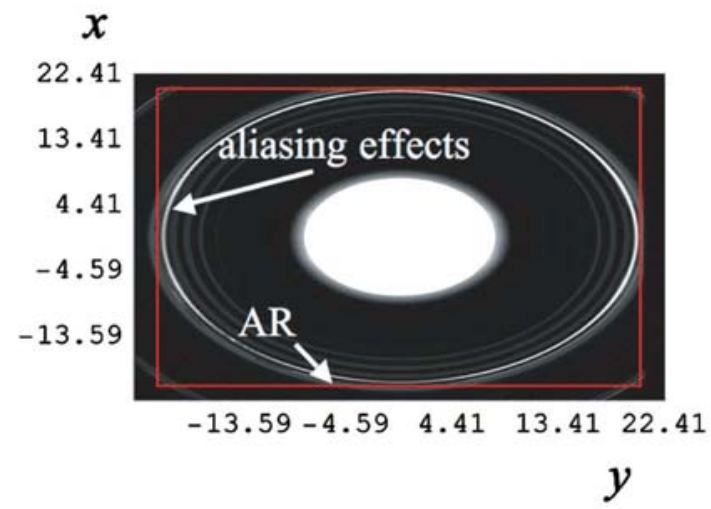

(b)

Fig. 6. (Color online) (a) Numerical results of $\left|U_{S}\right|$ for a circular aperture, with $t_{v}=0, z=0.1 \mathrm{~m}, L=0.1 \mathrm{~mm}$ over the range $-23 \mathrm{~mm}<x<23 \mathrm{~mm},-23 \mathrm{~mm}<y<23 \mathrm{~mm}$. (b) Contour plot of $\left|U_{S}\right|$ for a circular aperture, with $t_{v}=0 \mathrm{~s}, z=0.1 \mathrm{~m}$, and $L$ $=0.1 \mathrm{~mm}$ over the range $-23 \mathrm{~mm}<x<23 \mathrm{~mm},-23 \mathrm{~mm}<y$ $<23 \mathrm{~mm}$. Coordinates of the gray box (red online) defined in text, with $x_{\mathrm{SL}}=20.3126 \mathrm{~mm}$. AR, aliasing region.

present in the plot no longer span the range of 0 to the maximum value of $\sim 0.002$, but rather a lower maximum is chosen automatically that allows different features of the plot to be examined.]

Finally, in Figs. 7(a) and 7(b) we present contour plots of the diffraction patterns produced under the same conditions as Fig. 5; however, now we set $t_{v}$ equal to -0.5 $\times 10^{-12} \mathrm{~s}$ and $-1 \times 10^{-12} \mathrm{~s}$, respectively. We previously noted from Eq. (10) that the delimiting point $x_{\mathrm{SL}}$ is a function of $t_{v}$. On inspection of the two figures, we can see that as $t_{v}$ changes, so too does $x_{\mathrm{SL}}$ [and the gray box (red online)], accurately tracking the location where aliasing begins to occur.

\section{RELATING PULSE DISTRIBUTIONS AT DIFFERENT PLANES}

This section is concerned with a numerical redundancy that allows a significant reduction in the number of calculations that need to be performed if one wants to calculate the pulse distribution in two different $z$ planes. In the following discussion, an illustrative example is used to describe the technique. This section can be skipped without loss of continuity. 
$x$

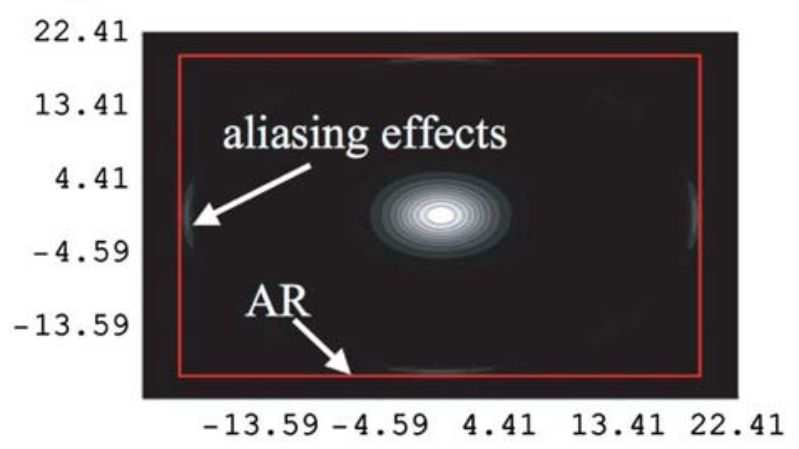

(a)

\section{$x$}

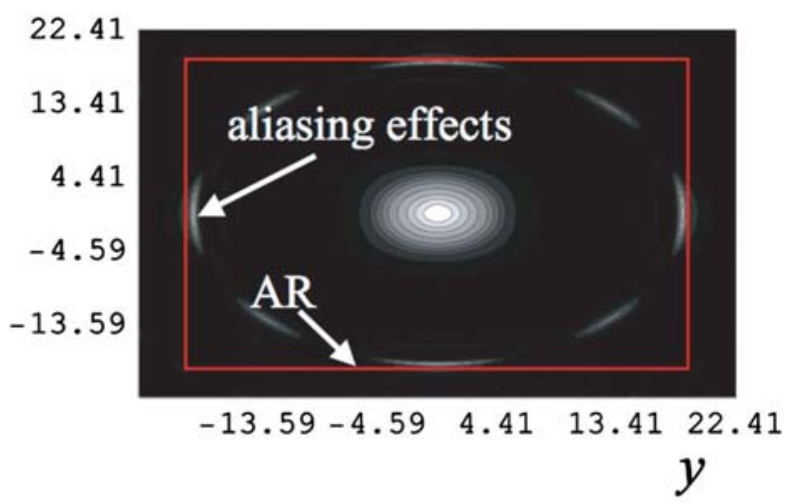

(b)

Fig. 7. (Color online) (a) Numerical results of $\left|U_{S}\right|$ for a square aperture, with $t_{v}=-0.5 \times 10^{-12} \mathrm{~s}, z=0.1 \mathrm{~m}$, and $L=0.1 \mathrm{~mm}$ over the range $-23 \mathrm{~mm}<x<23 \mathrm{~mm},-23 \mathrm{~mm}<y<23 \mathrm{~mm}$. Ten contour levels spanning 0 to $1.13 \times 10^{3}$. Coordinates of the gray box (red online) given by $x_{\mathrm{SL}}=19.564 \mathrm{~mm}$. AR, aliasing region. (b) Contour plot of $\left|U_{S}\right|$ for a square aperture, with $t_{v}=-1 \times 10^{-12} \mathrm{~s}$, $z=0.1 \mathrm{~m}$, and $L=0.1 \mathrm{~mm}$ over the range $-23 \mathrm{~mm}<x<23 \mathrm{~mm}$, $-23 \mathrm{~mm}<y<23 \mathrm{~mm}$. Ten contour levels spanning 0 to 3.78 $\times 10^{4}$. Coordinates of the gray box (red online) given by $x_{\mathrm{SL}}$ $=18.785 \mathrm{~mm}$. AR, aliasing region.

Consider the spectrum, $V_{2}(\omega)$, depicted in Fig. 4. We choose a suitable value for $\Delta \omega$ and sample the pulse at the discrete points indicated by the filled gray triangles in Fig. 4. By sampling, we generate a vector of $\omega$ values: $\omega_{n}^{\mathrm{I}}=\left[\omega_{1}, \omega_{2}, \ldots \omega_{N \omega}\right]$. To calculate the pulse distribution at the distance, $z_{1}=0.25 \mathrm{~m}$, we set $z=z_{1}$ and then define the $K$ vector using the relation: $K_{n}^{\mathrm{I}}=\left(2 \pi z_{1} c\right) / \omega_{n}^{\mathrm{I}}$. For each value of $K$ we now calculate and save (to the hard drive) a series of Fresnel diffraction patterns by successively implementing Eq. (1b). This calculation can be performed analytically if a known solution is available [20,26] or numerically with FFT-based techniques similar to those used for generating the results presented in Section 3 [22-25]. The pulse distribution at $z_{1}$ is then calculated from Eq. (4c) with $V_{2}\left(\omega_{n}^{\mathrm{I}}\right)$ and the vectors $K_{n}^{\mathrm{I}}$ and $\omega_{n}^{\mathrm{I}}$.

Suppose now that we wish to calculate the pulse distribution at a different $z$ plane, $z_{2}$, where $z_{2}>z_{1}$. The number of times we need to calculate Eq. (1b) for this pulse distribution can be reduced if we could reuse some of the previously calculated Fresnel diffraction patterns, defined by the vector $K_{n}^{\mathrm{I}}$. To do this we divide $K_{n}^{\mathrm{I}}$ by $z_{2}$ to produce a new $\omega$ vector; $\omega_{n}^{\mathrm{II}}$, plotted in Fig. 4 as filled black boxes.
Since $z_{2}>z_{1}$, the values of $\omega_{n}^{\mathrm{II}}$ must change, shifting to a higher frequency range than $\omega_{n}^{\mathrm{I}}$. This can be seen in Fig. 4 , where $\omega_{1}^{\mathrm{I}} \approx 100 \times 10^{12}$ but $\omega_{1}^{\mathrm{fl}} \approx 104.4 \times 10^{12}$ and where the values $\omega_{N_{\omega}}^{\mathrm{II}}$ and $\omega_{N_{\omega}-1}^{\mathrm{II}}$ now lie outside $\Delta \omega$. Also we note that the spacing between sampled points changes, i.e., $\left|\omega_{2}^{\mathrm{II}}-\omega_{1}^{\mathrm{II}}\right| \neq\left|\omega_{2}^{\mathrm{I}}-\omega_{1}^{\mathrm{I}}\right|$; however, any small change introduced in the sampling rate can be accounted for using Eq. (10). To calculate the pulse distribution at $z_{2}$, we need to calculate Eq. (1b) two more times (in this illustrative case), corresponding to the two new $K$ values indicated by the black

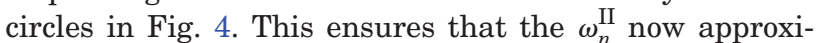
mately spans the same temporal frequency extent as $\omega_{n}^{\mathrm{I}}$. The pulse distribution at $z_{2}$ is then calculated from Eq. (4c) with $V_{2}\left(\omega_{n}^{\mathrm{II}}\right)$ and the vectors $K_{n}^{\mathrm{II}}$ and $\omega_{n}^{\mathrm{II}}$. It is important to note that even though the $z_{2}$ pulse calculation uses many of the same previously calculated Fresnel diffraction patterns (defined by the vector $K_{n}^{\mathrm{I}}$ ) as the $z_{1}$ calculation, each contributing Fresnel diffraction pattern is now weighted differently [22].

This approach certainly reduces the number of times that Eq. (1b) has to be calculated; however, one significant speed limitation is the time it takes to save and load calculated distributions to and from the hard drive into RAM. We believe this problem can be mitigated somewhat by using flash drives, which have faster read/write capabilities [22].

\section{STATIONARY PHASE ANALYSIS}

In this section we wish to examine in more detail the approximation made earlier in Section 2. In Section 2 we replaced the rectangular aperture $p(x)$ with a two-pinhole aperture $p_{T}(x)$, which simplified our diffraction problem. Despite the differences between the two solutions, it was found that the simplification made provided valuable insight into the diffraction process and ultimately led to the derivation of our sampling rules [Eq. (10a) and (10b)]. We begin this section by reexamining diffraction of a plane wave by a rectangular aperture. We proceed, closely following the analysis presented by Lohmann, see pp. 157162 of [36], and write the diffracted field (paraxial regime) $f_{z}(x, z)$ as

$$
f_{z}(x, z)=f_{0}(x) * h(x)
$$

where

$$
h(x)=\exp \left(\frac{j \pi x^{2}}{\lambda z}\right)
$$

and "*" indicates a convolution operation. We can rewrite Eq. (12a) as

$$
f_{z}(x, z)=\mathfrak{I}^{-1}\left\{F_{0}\left(f_{x}\right) H\left(f_{x}\right)\right\}(x),
$$

where

$$
F_{0}\left(f_{x}\right)=\mathfrak{I}\left\{f_{0}(x)\right\}\left(f_{x}\right)
$$

and

$$
H\left(f_{x}\right)=\Im\{h(x)\}\left(f_{x}\right)=\exp (j 2 \pi z / \lambda) \exp \left(-j \pi \lambda z f_{x}^{2}\right),
$$

with $\mathfrak{I}\{\Phi(x)\}\left(f_{x}\right)=\int_{-\infty}^{\infty} \Phi(x) \exp \left(-j 2 \pi f_{x} x\right) d x$ [19]. The $\mathfrak{I}$ and $\mathfrak{I}^{-1}$ operators used in this section differ from that used in 
Section 2 , in that they relate the spatial and spatial frequency representations, respectively, of a signal; see [19] for further clarification.

Since we are interested in diffraction from a rectangular aperture, we set $f_{0}(x)=p(x)$, thus

$$
\begin{aligned}
F_{0}\left(f_{x}\right)= & \int_{-L}^{L} \exp \left(-j 2 \pi x f_{x}\right) \mathrm{d} x \\
= & \frac{j \exp \left(-j 2 \pi f_{x} L\right)}{2 \pi f_{x}}-\frac{j \exp \left(j 2 \pi f_{x} L\right)}{2 \pi f_{x}}, \\
f_{z}(x, z)= & \exp (j 2 \pi z / \lambda) \int_{-\infty}^{\infty}\left[\frac{j \exp \left(-j 2 \pi f_{x} L\right)}{2 \pi f_{x}}\right. \\
& \left.-\frac{j \exp \left(j 2 \pi f_{x} L\right)}{2 \pi f_{x}}\right] \\
& \times \exp \left(-j \pi \lambda z f_{x}^{2}\right) \exp \left(j 2 \pi x f_{x}\right) \mathrm{d} f_{x}, \\
f_{z}(x, z)= & \exp (j 2 \pi z / \lambda)[Y(x-L)-Y(x+L)],
\end{aligned}
$$

where

$$
Y(x)=\int_{-\infty}^{\infty}\left(\frac{j}{2 \pi f_{x}}\right) \exp \left[j 2 \pi f_{x}\left(x-\frac{\lambda z f_{x}}{2}\right)\right] \mathrm{d} f_{x} .
$$

Although there is a singularity over the range of integration, we nevertheless apply the method of stationary phase to Eq. (15b) for reasons outlined on p. 158 of [36]. The phase of the kernel of Eq. (15b) can be written as

$$
\begin{aligned}
\phi\left(f_{x}\right) & =x f_{x}-\frac{\lambda z f_{x}^{2}}{2}, \\
\phi^{\prime}\left(f_{x}\right) & =\frac{\mathrm{d} \phi\left(f_{x}\right)}{\mathrm{d} f_{x}}=x-\lambda z f_{x} .
\end{aligned}
$$

Setting $\phi^{\prime}\left(f_{x}\right)=0$, we find the stationary point $f_{x s}=-x / \lambda z$. Now, using Eq. (13.20) on p. 115 of [36], we find the stationary phase approximation that $Y(x)$ is given by

$$
Y(x) \approx\left(\frac{-j \lambda z}{2 \pi}\right) \sqrt{\frac{\lambda}{\phi^{\prime \prime}(x)}} \exp (j \pi / 4)\left(\frac{1}{x}\right) \exp \left(\frac{j \pi x^{2}}{\lambda z}\right) .
$$

Substituting Eq. (17) back into Eq. (15b), we arrive at

$$
\begin{aligned}
f_{z}(x, z)= & \exp (j 2 \pi z / \lambda) \sqrt{\frac{\lambda}{\phi^{\prime \prime}(x)}} \exp (j \pi / 4)\left(\frac{j \lambda z}{2 \pi}\right) \\
& \times\left\{\Theta_{1}(x) \exp \left[\frac{j \pi(x-L)^{2}}{\lambda z}\right]\right. \\
& \left.+\Theta_{2}(x) \exp \left(\frac{j \pi(x+L)^{2}}{\lambda z}\right)\right\},
\end{aligned}
$$

where

$$
\Theta_{1}(x)=\frac{1}{L-x}, \quad \Theta_{2}(x)=\frac{1}{L+x} .
$$

The analysis presented here indicates that the points in the aperture plane that contribute most significantly to the diffraction integral are those just at the aperture edges, in line with the approximation made when deriving Eq. (8) in Section 2. Similar to Eq. (8b), Eq. (17) also consists of two emerging spherical waves located at $x$ $= \pm L$. There are differences between the two equations: scaling factors and the introduction of the terms $\Theta_{1}(x)$ and $\Theta_{2}(x)$. Nevertheless, this analysis provides some justification for the approximation derived in Section 2.

In Section 3, we presented numerical simulation results for a pulse diffraction from a circular aperture and found that the sampling rules seem to apply here as well. In this regard we wish to draw attention to the analysis presented by Stamnes in [37], particularly in Section 4 of his paper, where the diffraction of a plane wave by a circular aperture is shown to consist of both a geometrical optics component and a boundary diffraction wave that arises from the aperture edge. The main contributions to the boundary diffraction wave arise from two "critical points" located directly opposite each other on the edge of the circular aperture. We emphasis that the analysis presented here differs from that presented by Stamnes in that we do not specify a geometrical component; however, we do wish to note the similarity between the two cases, which seems to be validated by the numerical simulations presented in Section 3. In closing, we also point out the similarity of the analysis to that presented in [38] in relation to geometrical diffraction; see Section 3 in particular. Boundary wave diffraction has also been used to examine the diffraction of pulses [39].

\section{CONCLUSION}

In this paper we have examined sampling issues that arise when numerically simulating the diffraction of ultrashort pulses by apertures. Ultrashort pulses consist of many contributing spectral components, each of which must be considered in order to calculate the total diffraction pattern. Using an analytical expression to describe the diffraction of an individual spectral component, we examined how many of these components were needed so that a diffraction pattern free from numerical sampling errors could be calculated.

A simple set of guidelines were derived using a combination of graphical considerations and by simplifying the analytical expression describing the diffraction process. This simplification reduced the area of the contributing aperture from a rectangle to two point sources located at the aperture edges. This new expression was considerably easier to analyze and was used to develop the sampling guidelines presented in this work. In Section 3, these sampling rules were shown to hold for more general conditions than the circumstances under which they were derived. Later, in Section 5, we provided some justification for the approach adopted in Section 2 by examining the 
analytical diffraction integral using the method of stationary phase.

There are several important features of pulse propagation that are not addressed in this paper. We have, for example, considered propagation in free space only and therefore assumed a constant, wavelength-independent, refractive index. The different spectral components that make up a pulse, traveling, for example, in an optic fiber, "see" different refractive indices $[15,16]$. This will change sampling conditions derived here. Also we note that pulses with a positive or negative frequency chirp are not analyzed. We do not see any reason why these conditions cannot also be addressed using a framework similar to the one adopted here; however, more investigation would be required, which we feel is beyond the scope of the work presented here.

We do note that a sampling rule is presented in [15] stipulating that the phase difference between subsequent spectral components may not exceed $2 \pi$. We believe the analysis presented in this paper complements that in [15], providing an intuitive graphical picture that describes how undersampling the pulse results in numerical artifacts in the spatial domain. Related work on the propagation of partially coherent radiation is discussed in [40] and the references therein.

Finally, we add that the analysis presented in Section 5 was largely informed by [36]. In line with some other ideas presented in Lohmann's analysis, we could envisage a more complicated structure in the aperture plane. If this structure could be described using piecewise combination of slits of different widths, Eq. (8c) or Eq. (18) could be used as a basis for quickly (and approximately) guessing at the types of diffraction structures suitable for pulse shaping.

\section{ACKNOWLEDGMENTS}

This work was partly supported by the Austrian Scientific Fund FWF (SFB-ADLIS) and the EC (TERRANOVA, POISE).

\section{REFERENCES}

1. C. J. R. Sheppard, "Generalized Bessel pulse beams," J. Opt. Soc. Am. A 19, 2218-2222 (2002).

2. A. E. Kaplan, "Diffraction-induced transformation of nearcycle and subcycle pulses," J. Opt. Soc. Am. B 15, 951-956 (1998).

3. R. W. Ziolkowski, "Propagation characteristics of ultrawidebandwidth pulsed Gaussian beams," J. Opt. Soc. Am. A 9, 2021-2030 (1992).

4. G. P. Agrawal, "Spectrum-induced changes in diffraction of pulsed optical beams," Opt. Commun. 157, 52-56 (1998).

5. M. Gu, "Three-dimensional image formation in confocal microscopy under ultrashort laser pulse illumination," J. Mod. Opt. 42, 747-762 (1995).

6. M. Gu, Advanced Optical Imaging Theory, 1st ed. (Springer-Verlag, 2000).

7. M. A. Porras, "Ultrashort pulsed Gaussian light beams," Phys. Rev. E 58, 1086-1093 (1998).

8. C. F. R. Caron, "Free-space propagation of ultrashort pulses: spacetime couplings in Gaussian pulse beams," J. Mod. Opt. 45, 1881-1892 (1999).

9. C. J. R. Sheppard, "Bessel pulse beams and focus wave modes," J. Mod. Opt. 18, 2594-2600 (2001).
10. C. J. R. Sheppard and X. Gan, "Free-space propagation of femto-second light pulses," Opt. Commun. 133, 1-6 (1997).

11. M. Gu and X. S. Gan, "Fresnel diffraction by circular and serrated apertures illuminated with an ultrashort pulsedlaser beam," J. Opt. Soc. Am. A 13, 771-778 (1996).

12. A. Gürtler, C. Winnewisser, H. Helm, and P. Uhd Jepsen, "Terahertz pulse propagation in the near field and the far field," J. Opt. Soc. Am. A 17, 74-83 (2000).

13. M. Lefrancois and S. F. Pereira, "Time evolution of the diffraction pattern of an ultrashort laser pulse," Opt. Express 11, 1114-1122 (2003).

14. G. Girieud and S. F. Pereira, "Interference or not: analysis of Young's experiment for a single cycle pulse," J. Eur. Opt. Soc. Rapid Publ. 1, 06016-1 (2006).

15. S. P. Veetil, C. Vijayan, D. K. Sharma, H. Schimmel, and F. Wyrowski, "Sampling rules in the frequency domain for numerical propagation of ultrashort pulses through linear dielectrics," J. Opt. Soc. Am. B 23, 2227-2236 (2006).

16. S. P. Veetil, H. Schimmel, F. Wyrowski, and C. Vijayan, "Wave optical modelling of focusing of an ultra short pulse," J. Mod. Opt. 53, 2187-2194 (2006).

17. R. Gase, "Ultrashort-pulse measurements applying generalized time-frequency distribution functions," J. Opt. Soc. Am. B 14, 2915-2920 (1997).

18. C. Dorrer and I. Kang, "Complete temporal characterization of short optical pulses by simplified chronocyclic tomography," Opt. Lett. 28, 1481-1483 (2003).

19. J. O'Hara and D. Grischkowsky, "Quasi-synthetic phasedarray terahertz imaging,” J. Opt. Soc. Am. B 21, 1178-1191 (2004).

20. J. W. Goodman, Introduction to Fourier Optics, 3rd ed. (Roberts \& Co., 2005).

21. E. Hecht, Optics, 2nd edition (Addison-Wesley, 1989).

22. D. P. Kelly, B. M. Hennelly, A. Grün, J. Darmo, and K. Unterrainer, "Fast numerical algorithm for ultrashort $\mathrm{THz}$ pulse diffraction," Proc. SPIE 6697, 66970Q (2007).

23. B. M. Hennelly and J. T. Sheridan, "Generalizing, optimizing, and inventing numerical algorithms for the fractional Fourier, Fresnel, and linear canonical transforms,” J. Opt. Soc. Am. A 22, 917-927 (2005).

24. B. M. Hennelly and J. T. Sheridan, "Optical encryption and the space bandwidth product," Opt. Commun. 247, 291-305 (2005).

25. D. P. Kelly, B. M. Hennelly, W. T. Rhodes, and J. T. Sheridan, "Analytical and numerical analysis of linear optical systems," Opt. Eng. (Bellingham) 45, 088201 (2006).

26. D. P. Kelly, J. T. Sheridan, and W. T. Rhodes, "Finiteaperture effects for Fourier transform systems with convergent illumination. Part I: 2-D system analysis," Opt. Commun. 263, 171-179 (2006).

27. R. N. Bracewell, The Fourier Transform and Its Applications (McGraw-Hill, 1965).

28. M. Unser, "Sampling-50 years after Shannon," Proc. IEEE 88, 569-587 (2000).

29. A. Stern and B. Javidi, "Sampling in the light of Wigner distribution," J. Opt. Soc. Am. A 21, 360-366 (2004).

30. A. Stern and B. Javidi, "Sampling in the light of Wigner distribution: errata," J. Opt. Soc. Am. A 21, 2038-2038 (2004).

31. K. B. Wolf, D. Mendlovic, and Z. Zalevsky, "Generalized Wigner function for the analysis of superresolution systems," Appl. Opt. 37, 4374-4379 (1998).

32. D. Mendlovic and A. W. Lohmann, "Space-bandwidth product adaptation and its application to superresolution: fundamentals," J. Opt. Soc. Am. A 14, 558-562 (1997).

33. D. Mendlovic, A. W. Lohmann, and Z. Zalevsky, "Space-bandwidth product adaptation and its application to superresolution: examples," J. Opt. Soc. Am. A 14, 563-567 (1997).

34. G. B. Thomas, Jr., and R. L. Finney, Calculus (AddisionWesley, 1996).

35. S. Wolf, The Mathematica Book, 4th ed. (Cambridge U. Press, 1999). 
36. A. W. Lohmann, Optical Information Processing, S. Sinzinger, ed. (Universitätsverlag Ilmenau, 2006).

37. J. Stamnes, "Waves, rays and the method of stationary phase," Opt. Express 10, 740-751 (2002).

38. J. B. Keller, "Geometrical theory of diffraction," J. Opt. Soc. Am. 52, 116-130 (1962)

39. Z. L. Horvath and Zs. Bor, "Diffraction of short pulses with boundary diffraction wave theory,” Phys. Rev. E 63, 026601 (2001).

40. C. Rydberg and J. Bengtsson, "Efficient numerical representation of the optical field for the propagation of partially coherent radiation with a specified spatial and temporal coherence function," J. Opt. Soc. Am. A 23, 1616-1625 (2006). 